\title{
Patent ductus arteriosus (PDA) staging and splanchnic circulation in high risk preterm infants
}

\begin{abstract}
Objective: To evaluate the influence of PDA on superior mesenteric artery (SMA) and celiac artery (CA) in the immediate postnatal period in preterm infants.

Methods: 50 preterm less than 32 week with birth weight less than $1 \mathrm{~kg}$ was included in the prospective observation study. PDA (PSV: peak systolic velocity, EDV: end diastolic velocity, TAV: time average velocity). Blood flow indices (resistive index; RI, pulsatility index; PI) was calculated. PDA was categorized as small, moderate and large. Values taken at $48 \mathrm{hr}$ were considered for statistical calculation.

Result: Significant PDA was associated with high SMA PSV and low EDV in both SMA and CA. Large $(n=12)$ and moderate PDA $(n=3)$ was associated with high PSV and low EDV in SMA, hence was associated with significantly high RI (large; $0.85 \pm 0.04$, moderate; $0.80 \pm 0.03)$ in mesenteric circulation. In celiac circulation, large PDA was associated with low EDV (11.66 \pm 6.09$)$ and moderate PDA was associated with significant high PSV $(64.33 \pm 13.05)$. This velocity pattern in CA was associated with significant high RI $(0.77 \pm 0.08)$ and high PI $(1.73 \pm 0.52)$ in large PDA group. Velocities in small PDA and no PDA groups were comparable in both arteries.

Conclusion: Significant PDA was associated with attenuated blood velocities in SMA compromising the intestinal perfusion. Celiac artery blood flow velocities were adversely affected by large PDA. Low EDV is the predicting factor for compromised perfusion in both the arteries. PDA diameter and flow in descending aorta was the determining factor.
\end{abstract}

Keywords: preterm infants, patent ductus arteriosus, superior mesenteric artery, celiac artery
Volume 9 Issue 4 - 2019

\author{
Khaled El Atawi,' Nitin Unde, ${ }^{2}$ Mahmoud \\ Elhalik ${ }^{3}$ \\ 'Latifa women and children hospital, Neonatal intensive care \\ unit, Dubai, UAE \\ ${ }^{2}$ Latifa women and children hospital, Neonatal intensive care \\ unit, Dubai, UAE \\ ${ }^{3}$ Latifa women and children hospital, Neonatal intensive care \\ unit, Dubai, UAE
}

Correspondence: Dr Khaled El-Atawi, Latifa women and children hospital, Neonatal intensive care unit, Dubai health authority, UAE, Email kelatawi@eim.ae

Received: January 07, 2019 | Published: July 29, 2019

\section{Introduction}

Patent ductus arteriosus (PDA) is a common complication in preterm infants, especially in extremely low birth weight (ELBW) infants with an incidence of $80-90 \% .{ }^{1}$ The current body of evidence showed a significant association between PDA and neonatal mortality and morbidities, mainly due the presence of left to right shunt -ductal steal phenomena- and consequent hypoperfusion to vital organs., ${ }^{2,3}$ However, there is a growing controversy about the role of aggressive therapeutic interventions in the management of PDA, previous reports indicated that $73 \%$ of preterm infants with PDA will show spontaneous closure of the duct by the end of first week and that the beneficial role of medical/surgical treatment is questionable., Thus, it is important to identify and discriminate infants with hemodynamically significant ductus arteriosus (hsPDA). A growing body of evidence has investigated the role of several methods in the identification of hsPDA. Biomarkers, as natriuretic peptides and ischemia-modified albumin, were reported to significantly correlated with early hsPDA and have a good predictive value. ${ }^{6,7}$ Other reports showed high diagnostic accuracy of clinical scores for the detection of hsPDA infants who needs treatment. ${ }^{8}$ However, there is no consensus about a grading system for hsPDA; functional echocardiography is considered as gold standard in the early diagnosis of significant PDA and focus on early targeted treatment. ${ }^{9}$ McNamara \& Sehgal, ${ }^{10}$ proposed a staging system with a comparison between clinical and echocardiographic criteria of hsPDA.

On the other hand, hsPDA was reported to be associated with a significant reduction in regional blood flow, hsPDA reduced blood flow velocity (BFV) in the cerebral, renal, and mesenteric arteries. $^{11,12}$ Freeman-Ladd and colleagues ${ }^{13}$ noted a significant absence or reversal of diastolic flow in the superior mesenteric artery (SMA) among preterm infants with hsPDA. Another report showed that retrograde diastolic flow in the descending aorta may act as a potential marker of high-volume shunt and hypoperfuion of lower body segment. ${ }^{14}$ Additionally, SMA and celiac artery (CA) BVF increased dramatically after ductal closure in ELBW infants, which may predispose to oxidative stress and reperfusion injury of the gut. ${ }^{15}$ Therefore, the hemodynamic effects of hsPDA on the SMA and CA blood flow may play critical role in targeted treatment strategies. Our aim was to do the accurate estimation of blood flow velocities in both SMA and CA in high risk infants with PDA with a cohort. This relationship may provide an accurate estimate of compromise to intestinal circulation due to duct and provide a rationale for treatment to reduce the intestinal morbidity.

\section{Methods}

\section{Subjects}

a. Infants admitted to tertiary neonatal intensive care unit with GA less than 32 week and birth weight less than $1 \mathrm{~kg}$ were enrolled for the study. Exclusion criteria were major congenital malformations, critical congenital heart disease and evidence of perinatal asphyxia requiring significant resuscitation (need of drugs).

b. The demographic data of study population is shown in Table 1 . 
c. This study was approved by Dubai health authority (DHA) ethical committee and written informed consent was obtained from the parents before the subject enrollment.

\section{Study design}

50 infants fulfilling the inclusion criteria were included in this prospective trial, who underwent bedside ultrasound twice at $24 \mathrm{hr}$ Table I Subject characteristics of the study population (50 new-born)
(20-30) and 48hr (40-54) to measure the blood flow velocities in SMA and CA along with simultaneous assessment of PDA. The measurements were done by single certified neonatologist trained in functional echocardiography and data for various factors was collected simultaneously (table 2). We utilized non-probability, consecutive, sampling technique to recruit eligible infants.

\begin{tabular}{|c|c|c|c|c|c|}
\hline \multicolumn{6}{|l|}{ PDA category (total no $=50$ ) } \\
\hline Subject characteristics & $\begin{array}{l}\text { Large PDA } \\
(\mathrm{N}=12)\end{array}$ & $\begin{array}{l}\text { Moderate PDA } \\
(\mathrm{N}=3)\end{array}$ & $\begin{array}{l}\text { Small PDA } \\
(\mathrm{N}=22)\end{array}$ & $\begin{array}{l}\text { NO PDA } \\
(\mathrm{N}=13)\end{array}$ & $P$ value \\
\hline Birth weight(gm) & $854.25 \pm 103.24$ & $788.33 \pm 212.74$ & $844.68 \pm 157.13$ & $846.15 \pm 187.56$ & 0.93 \\
\hline Gestational age(weeks) & $26.83 \pm 1.46$ & $25.33 \pm 1.15$ & $27.0 \pm 2.46$ & $27.15 \pm 2.19$ & 0.61 \\
\hline Gender ( $\%$ male) & 33.3 & 33.3 & 63.6 & 53.8 & 0.34 \\
\hline Delivery method (\% C-section) & 75.0 & 33.3 & 77.3 & 69.2 & 0.45 \\
\hline \multicolumn{6}{|l|}{ Clinical measures } \\
\hline Mean blood pressure (mmhg) & $35.5 \pm 7.90$ & $35.33 \pm 4.16$ & $38.55 \pm 9.11$ & $42.00 \pm 7.23$ & 0.23 \\
\hline Haematocrit (\%) & $45.58 \pm 6.24$ & $42.67 \pm 4.6 I$ & $46.45 \pm 6.26$ & $45.46 \pm 6.96$ & 0.80 \\
\hline Duration to full feeds & $25.43 \pm 10.83$ & $33.50 \pm 6.36$ & $24.38 \pm 14.32$ & $27.11 \pm 19.87$ & 0.868 \\
\hline$(n=34)$ & $(N=7)$ & $(N=2)$ & $(N=16)$ & $(N=9)$ & \\
\hline \multicolumn{6}{|l|}{ Confounding factors } \\
\hline Umbilical artery catheter (\%) & 25 & 33.3 & 18.2 & 23.1 & 0.98 \\
\hline Caffeine (\%) & 100 & 100 & 100 & 100 & - \\
\hline Antenatal steroid 2 doses (\%) & 58.3 & 33.3 & 59.1 & 38.5 & 0.57 \\
\hline
\end{tabular}

Values showing percentage of patients are labelled with \% sign. Otherwise, values are reported as means and SDs, mean \pm SD

Table 2 SMA blood flow velocities

\begin{tabular}{|c|c|c|c|c|c|}
\hline $\begin{array}{l}\text { SMA } \\
\text { Variables }\end{array}$ & $\begin{array}{l}\text { Large PDA } \\
(\mathrm{N}=\mid 2)\end{array}$ & $\begin{array}{l}\text { Moderate PDA } \\
(\mathrm{N}=3)\end{array}$ & Small PDA ( $N=22)$ & $\begin{array}{l}\text { NO PDA } \\
(\mathrm{N}=13)\end{array}$ & $P$ value \\
\hline $\mathrm{PSV}(\mathrm{cm} / \mathrm{s})$ & $45.62 \pm 13.68$ & $38.50 \pm 9.96$ & $38.27 \pm 10.88$ & $37.65 \pm 6.47$ & 0.219 \\
\hline $\mathrm{EDV}(\mathrm{cm} / \mathrm{s})$ & $6.2 I \pm 2.71$ & $6.83 \pm 0.76$ & $8.61 \pm 3.61$ & $7.95 \pm 1.74$ & 0.148 \\
\hline $\operatorname{TAV}(\mathrm{cm} / \mathrm{s})$ & $18.50 \pm 7.55$ & $17.0 \pm 2.59$ & $16.05 \pm 5.02$ & $15.38 \pm 3.05$ & 0.485 \\
\hline RI & $0.85 \pm 0.04$ & $0.80 \pm 0.03$ & $0.77 \pm 0.04$ & $0.77 \pm 0.05$ & $0.000 * *$ \\
\hline PI & $2.11 \pm 0.25$ & $1.78 \pm 0.42$ & $1.83 \pm 0.32$ & $1.76 \pm 0.40$ & 0.058 \\
\hline
\end{tabular}

$* \mathrm{P}<0.05, * * \mathrm{p}<0.01$

Data are mean \pm SD unless otherwise indicated

Abbreviations: PSV, peak systolic velocity; EDV, end-diastolic velocity; TAV, time-averaged mean velocity; RI, resistive index; PI, pulsatile index; PDA, patent ductus arteriosus; SMA, superior mesenteric artery 


\section{Doppler ultrasound studies and evaluation of PDA:}

a. SMA and CA BFV were measured using pulsed Doppler ultrasound (vivid q GE, USA) and a $10 \mathrm{MHz}$ transducer. A realtime two-dimensional image and colour flow mapping was used to identify the arteries. SMA was identified as the second major branch of the abdominal aorta, originating just below the CA. The sampling volume of the pulsed Doppler was placed $3 \mathrm{~mm}$ distal to the origin of the SMA and CA using a real-time two-dimensional image from a longitudinal abdominal approach. Angle correction was used when necessary.

b. When stable waveforms measurements were obtained, the curves were traced and the blood flow variables in each artery were calculated from at least three consecutive cardiac cycles of optimal quality .The recorded blood flow variables were the peak systolic velocity (PSV), end-diastolic velocity (EDV), time-averaged mean velocity (TAV). At least two sets measurements were taken at each time point, and the mean of these readings was used for the final analysis. The resistance index (RI): (PSV-EDV)/PSV. The pulsatility index (PI): ( PSV-EDV)/TAV

c. Assessment of PDA was done by measurement of PDA size, LA to aortic ratio and descending aortic end diastolic flow. PDA was defined as small, moderate and large on the basis of above variables.

A. Ductal diameter: ductal diameter $\geq 1.5 \mathrm{~mm}$ was considered significant. ${ }^{7,8}$

Large :> 3mm, moderate $1.5-3 \mathrm{~mm}$, small $<1.5 \mathrm{~mm}$

B. LA: Ao: we used a cut-off ratio of $\geq 1.5$ to define significance. ${ }^{7,8}$

Large: $>2$, moderate $1.5-2$, small $<1.5$

C. Descending aortic end diastolic velocity: absent or reversed end diastolic flow. ${ }^{8.9}$

Large: reversal DA flow, Moderate: absent DA flow, small: normal DA flow

\section{Statistical analysis}

Blood flow variables readings at $48 \mathrm{hr}$, were compared using ANOVA test for different PDA groups. Subject characteristics and clinical variables were recorded for four PDA groups: large, moderate, small and no PDA. ANOVA and chi-square test were used to detect statistically significant difference (alpha $=0.05$ ) based on continuous or categorical nature of the item. Statistical analysis was performed by using SPSS (statistical Package for Social Sciences) version 20.0.

\section{Result}

Fifty infants were enrolled. The mean birth weight and GA of enrolled patients was $844 \pm 153 \mathrm{gm}$ and $27 \pm 2.1$ week respectively. Out of the total study population, 15 infants were diagnosed with significant PDA (large: 12, moderate: 3 ) as per the predefined echocardiography criteria. table 1 summarizes the demographic and clinical data. Birth weight and gestational age were similar between the groups, and the mean blood pressure values were low in the significant PDA group. Caffeine, hematocrit and presence of UAC (known modifiers of SMA and CA blood flow velocities) did not differ between the groups. 34 infants were followed for duration to reach full feeds, which was not influenced by the type of PDA. Large PDA was associated with high PSV and low EDV, whereas moderate PDA was associated with low EDV in superior mesenteric artery circulation. This flow pattern was associated with significantly high SMA RI (large $0.85 \pm 0.04$, moderate $0.80 \pm 0.03$ ) and PI (large 2.11 \pm 0.25 ) in significant PDA groups compared to others. SMA blood flow velocities (Table 2) and indicies were comparable between small and no PDA groups. SMA TAV was not influenced by PDA type. Neonates with large PDA had low EDV (11.66 \pm 6.09$)$ and moderate PDA had significantly high PSV $(64.33 \pm 13.05)$ compared to other groups in celiac artery circulation. The velocity pattern in CA (Table 3 ) was associated with significantly high RI $(0.77 \pm 0.08)$ and high PI $(1.73 \pm 0.52)$ in large PDA infants compared to other three groups. CA RI and PI in moderate PDA group were comparable with small/no PDA groups. CA TAV was comparable in all PDA groups.

Table 3 CA blood flow velocities

\begin{tabular}{|c|c|c|c|c|c|}
\hline CA variables & $\begin{array}{l}\text { Large PDA } \\
(\mathbf{N}=\mid 2)\end{array}$ & $\begin{array}{l}\text { Moderate PDA } \\
(\mathrm{N}=3)\end{array}$ & Small PDA (N=22) & $\begin{array}{l}\text { NO PDA } \\
(\mathbf{N}=\mid 3)\end{array}$ & P value \\
\hline $\operatorname{PSV}(\mathrm{cm} / \mathrm{s})$ & $56.70 \pm 13.56$ & $64.33 \pm 13.05$ & $47.35 \pm 10.65$ & $46.76 \pm 11.36$ & $0.022^{*}$ \\
\hline $\operatorname{EDV}(\mathrm{cm} / \mathrm{s})$ & $11.66 \pm 6.09$ & $19.66 \pm 4.75$ & $|4.2| \pm 5.12$ & $15.65 \pm 4.18$ & 0.076 \\
\hline $\operatorname{TAV}(\mathrm{cm} / \mathrm{s})$ & $26.87 \pm 6.77$ & $36.66 \pm 8.80$ & $25.97 \pm 9.46$ & $26.73 \pm 7.35$ & 0.237 \\
\hline RI & $0.77 \pm 0.08$ & $0.68 \pm 0.01$ & $0.69 \pm 0.07$ & $0.65 \pm 0.05$ & $0.001 * *$ \\
\hline PI & $1.73 \pm 0.52$ & $1.20 \pm 0.10$ & I. $37 \pm 0.29$ & $|| 7 \pm 0.21$. & $0.001 * *$ \\
\hline
\end{tabular}

$* \mathrm{p}<0.05, * * \mathrm{p}<0.01$

Data are the mean \pm SD unless otherwise indicated.

Abbreviations: PSV, peak systolic velocity; EDV, end-diastolic velocity; TAV, time-averaged mean velocity; RI, resistive index; PI, pulsatile index; PDA, patent ductus arteriosus; CA, celiac artery 


\section{Discussion}

Despite widespread use of the term, there is no consensus on the definition of hemodynamically significant PDA. The echocardiography criteria used in the literature to define HsPDA includes: ${ }^{2,10,11}$ Diameter $>1.5 \mathrm{~mm}$ at the narrowest point, absent or retrograde diastolic flow in descending aorta and major arteries( superior mesenteric and anterior cerebral artery),left atrial and aorta ratio $>1.5$ and unrestrictive pulsatile flow PDA pattern. Considering all the previous reports, we used PDA diameter, flow in DA and LA: RO to define the PDA. Infant's characteristics, possibly affecting intestinal perfusion, were similar among groups. Recorded mean blood pressure reading was low in significant PDA group compared to other, as reported by Evan et al ${ }^{12}$. This can be explained by the ductal steal associated with significant PDA.

Left to right shunting across a PDA is associated with systemic hypoperfusion. Echocardiography assessment of the celiac and superior mesenteric arteries demonstrates reduced blood flow in the presence of a PDA compared to controls. ${ }^{13-15}$ This may explain the association between PDA and NEC. Coombs et al., ${ }^{16}$ reported abnormal SMA velocities associated with PDA despite increase in left ventricular output. Studies have reported abnormal diastolic blood flow in major arteries including SMA in infants with HsPDA. ${ }^{5,6}$ Frank et al., ${ }^{17}$ demonstrated high PSV and negative EDV in preterm with significant PDA and concluded that increase SMA PSV with PDA is a compensatory mechanism to maintain the intestinal perfusion. Similarly Deeg et al., ${ }^{18}$ reported significantly low EDV in newborn with PDA leading to compromised gut perfusion. Confirming previous reports, our study showed abnormal SMA BFV associated with significant PDA. We also demonstrated the influence on SMA and CA velocities as per the severity of significant PDA (large/ moderate). Large PDA influences both PSV (high) and EDV (low) compared to moderate PDA, which is associated with low EDV. This pattern showed low EDV is the determining factor for compromised intestinal perfusion in high risk preterm infants with significant PDA, which indicates diastolic run-off.

EL-Khuffash et al. ${ }^{14}$ reported low celiac artery blood flow (CAF) in presence of significant PDA in spite of increased left ventricular output (LVO), had a sensitivity of $93 \%$ and specificity of $78 \%$.We reported large PDA is associated with adverse CA BFV compared to moderate PDA. Large PDA influences both PSV (high) and EDV (low) compromising the intestinal perfusion. Comparable velocity pattern in both SMA and CA associated with small and no PDA group showed similar outcome as reported previously. ${ }^{19}$ Shimada et al., 5 reported lower splanchnic velocities (SMA and CA) with significant PDA in extreme low birth weight preterm. This abnormal blood flow pattern in SMA and CA reflected by the increased resistance indexes in the infants with hsPDA is consistent with ductal steal phenomenon (diastolic shunt from aorta to main pulmonary artery). ${ }^{20,21}$ Clyman et al. ${ }^{22}$ have shown that the decreased splanchnic and renal blood flows in preterm lambs with large left to right PDA was a consequence of both the ductal steal and localized increase in vascular resistance.

This study shows the exact influence of PDA staging on both the SMA and CA BFV in high risk preterm population. This blood flow pattern helps the clinician to limit the feeding related complications during the early postnatal life in preterm infants with significant PDA. We acknowledge that the present study has some limitations. The study was a single- center experience with relatively small sample size which may affect the generalizability of our findings. Moreover, it was not possible to evaluate all the echocardiography parameters used to quantify the hemodynamic consequences of significant PDA.

\section{Conclusion}

Both large and moderate PDA was associated with abnormal velocity pattern in SMA causing compromised intestinal perfusion. On the contrary, only large PDA was associated with compromised celiac artery perfusion. Low EDV was the determining factor for the abnormal velocity pattern in both the arteries. PDA diameter and abnormal DA flow determines the flow pattern.

\section{Acknowledgments}

Written informed consent was obtained from the parents before the subject enrollment. This study was approved by Dubai health authority (DHA) ethical committee.

\section{Conflicts of interest}

The authors have no conflicts of interest to declare.

\section{Funding}

None.

\section{References}

1. Van Overmeire B, Chemtob S. The pharmacologic closure of the patent ductus arteriosus. Semin Fetal Neonatal Med. 2005;10(2):177-184.

2. Skelton R, Evans N, Smythe J. A blinded comparison of clinical and echocardiographic evaluation of the preterm infant for patent ductus arteriosus. J Paediatr Child Health. 1994;30(5):406-411.

3. Alagarsamy S, Chhabra M, Gudavalli M, et al. Comparison of clinical criteria with echocardiographic findings in diagnosing PDA in preterm infants. J Perinat Med. 2005;33(2):161-164.

4. Clyman RI. Mechanisms regulating the ductus arteriosus. Biol Neonate. 2006;89(4):330-335

5. Shimada S, Kasai T, Hoshi A, et al. Cardiocirculatory effects of patent ductus arteriosus in extremely low-birth-weight infants with respiratory distress syndrome. Pediatr Int. 2003;45(3):255-262.

6. Swarup J, Baker RW, Brozanski BS, et al. Asymmetry of cerebral blood flow velocity in low birth weight infants. Biol Neonate. 2005;87(3):145151.

7. El Hajjar M, Vaksmann G, Rakza T, et al. Severity of the ductal shunt: a comparison of different markers. Arch Dis Child Fetal Neonatal Ed. 2005;90:F419-422.

8. Sehgal A, McNamara PJ. Does echocardiography facilitate determination of hemodynamic significance attributable to the ductus arteriosus? Eur $J$ Pediatr. 2009;168(8):907-914

9. Kluckow M, Evans N. Early echocardiographic prediction of symptomatic patent ductus arteriosus in preterm infants undergoing mechanical ventilation. J Pediatr. 1995;127(5):774-779.

10. Kluckow M, Jeffery M, Gill A, et al. A randomised placebo-controlled trial of early treatment of the patent ductus arteriosus. Arch Dis Child Fetal Neonatal Ed. 2014;99(2):99-104.

11. McNamara PJ, Sehgal A. Towards rational management of the patent ductus arteriosus: the need for disease staging. Arch Dis Child Fetal Neonatal Ed. 2007;92:424-427. 
12. Evans N, Moorcraft J. Effect of patency of the ductus arteriosus on blood pressure in very preterm infants. Arch Dis Child. 1992;67:1169-117.

13. El-Khuffash A, Higgins M, Walsh K, et al. Quantitative assessment of the degree of ductal steal using celiac artery blood flow to left ventricular output ratio in preterm infants. Neonatology. 2008;93(3):206-212.

14. Ichihashi K, Shiraishi H, Endou H. Cerebral and abdominal arterial hemodynamic in preterm infants with patent ductus arteriosus. Acta Paediatr Jpn.1990;32(4):349-56.

15. Freeman-Ladd M, Cohen JB, Carver JD, et al. The hemodynamic effects of neonatal patent ductus arteriosus shunting on superior mesenteric artery blood flow. J Perinatol. 2005;25(7):459-462.

16. Coombs RC, Morgan MEI, Durbin GM, et al. Abnormal gut blood flow velocities in neonates at risk of necrotizing enterocolitis. J Pediatr Gastr Nutr. 1992;15(1):13-19.

17. Frank B, Jaap Schipper, Margotvan de Bor. Blood velocity wave form characteristics of superior mesenteric artery and anterior cerebral artery before and after ductus arteriosus closure. European Journal of Ultrasound. 1995;2(3):183-189.
18. Deeg KH, Gerstner R, Bundscherer F, et al. Doppler sonographic detection of reduced flow velocity in the celiac trunk of the newborn infant with patent ductus arteriosus. Monatsschr Kinderheilkd. 1987;135(1):24-29.

19. Sellmer A, Bjerre JV, Schmidt MR, et al. Morbidity and mortality in preterm neonates with patent ductus arteriosus on day 3. Arch Dis Child Fetal Neonatal Ed. 2013;98(6):F505-F510.

20. Wong SN, Lo RN, Hui PW. Abnormal renal and splanchnic arterial Doppler pattern in premature babies with symptomatic patent ductus arteriosus. $J$ Ultrasound Med. 1990;9(3):125-130.

21. Bomelburg T, Jorch G. Abnormal blood flow patterns in renal arteries of small preterm infants with patent ductus arteriosus detected by Doppler ultrasonography. Eur J Pediatr. 1989;148(7):660-664.

22. Clyman RI, Mauray F, Heymann MA, et al. Cardiovascular effects of patent ductus arteriosus in preterm lambs with respiratory distress. $J$ Pediatr. 1987;111(4):579-587. 